\title{
Editorial
}

\section{Scientific Programming towards a Smart World}

\author{
Wenbing Zhao, ${ }^{1}$ Xiong Luo, ${ }^{2}$ HuaPing Liu, ${ }^{3}$ Kun Hua, ${ }^{4}$ and Chaomin Luo ${ }^{5}$ \\ ${ }^{1}$ Cleveland State University, Cleveland, OH, USA \\ ${ }^{2}$ University of Science and Technology Beijing, Beijing, China \\ ${ }^{3}$ Tsinghua University, Beijing, China \\ ${ }^{4}$ Lawrence Technological University, Southfield, MI, USA \\ ${ }^{5}$ University of Detroit Mercy, Detroit, MI, USA \\ Correspondence should be addressed to Wenbing Zhao; w.zhao1@csuohio.edu
}

Received 26 February 2017; Accepted 27 February 2017; Published 20 March 2017

Copyright (C) 2017 Wenbing Zhao et al. This is an open access article distributed under the Creative Commons Attribution License, which permits unrestricted use, distribution, and reproduction in any medium, provided the original work is properly cited.

In the past several years, we have seen dramatic advancement in many application domains enabled by the integration of Internet of Things and cloud computing, computational intelligence algorithms, and soft computing methodologies. This development has resulted in many emerging highly multidisciplinary research areas typically termed as smart- ${ }^{*}$ technologies and systems, including smart-healthcare, smarthome, and smart-grid, as well as smart vehicles and intelligent transportation systems. These new technologies are transforming our society towards a smart world.

This special issue attracted 32 high quality submissions from countries around the globe, including China, USA, UK, Korea, Saudi Arabia, Greece, and Algeria. After a rigorous review process, 18 papers were accepted in this issue. The research presented in these papers broadly covers the interesting scope of this special issue, including computer networking (3 papers), intelligent transportation and navigation (3 papers), computer vision ( 2 papers), knowledge discovery (2 papers), scientific computing (2 papers), cloud computing ( 2 papers), gesture recognition and authorization (2 papers), and Internet of Things and machine learning (1 each).

In the area of computer networking, X. Yang et al. proposed a novel localization algorithm based on Voronoi diagram and rank sequence in wireless sensor networks; X. Liu et al. introduced a new program code dissemination scheme (referred to as FPCD) for wireless software defined networks; and K.-H. Kim et al. reported a cascade control based method for queue management in smart-grid IP networks.

In the areas of intelligent transportation and navigation, M. Dong et al. described a sophisticated navigation method using sonar point cloud data for underwater remotely operated vehicles; Y. Xing et al. proposed a new traffic flow forecasting method based on extreme learning machine; and $\mathrm{H}$. Gao et al. proposed a novel lateral control method for intelligent vehicles.

On computing vision research, $\mathrm{H}$. Wang et al. introduced a robust automatic target recognition algorithm for large scene images, and Y. Li et al. reported a novel approach for scene reconstruction based on metric online monocular SLAM.

On the topic of cloud computing, Y. Lu et al. proposed a new workload forecasting model for cloud services, which is based on random variable learning rate backpropagation neural network, and $\mathrm{X}$. Li et al. introduced a novel resource allocation mechanism for cloud workflow systems, where the resource allocation problem is treated as a market-oriented reverse auction problem.

On human gesture related research, B. Fang et al. provided a comprehensive report on how to use magnetic and inertial measurement units to accurately recognize hand and arm gestures, and A. M. H. Wong and D.-K. Kang proposed a novel stationary hand gesture authentication scheme using edit distance on finger pointing direction interval. 
This issue also includes two papers on knowledge discovery. Y. Zhu et al. reported a research on using neural network based machine learning methods to build a geological knowledge graph. X. Han et al. described how to use fragment based semantic graph model to perform text summarization.

In addition, this special issue includes exciting works on using computer programming algorithms to help solve science problems. P. Shi et al. introduced a novel experiment platform for materials correction study. The throughput of the platform was significantly improved by using state of the art computing vision algorithms. Furthermore, X. Wang et al. proposed an efficient and simple rigid-fluid coupling scheme with programming algorithms for particle-based fluid simulation and visualization.

Finally, Z. Liu et al. proposed a novel sparse representation method for the class-imbalance problem. Last, but not least, L. Ding et al. reported a very interesting system based on Internet of Things that can be used to monitor dangerous goods in container yards.

\section{Acknowledgments}

The guest editors would like to thank the authors for contributing to this special issue and thank all the reviewers for their time and rigorous reviews.

Wenbing Zhao

Xiong Luo

HuaPing Liu

Kun Hua

Chaomin Luo 

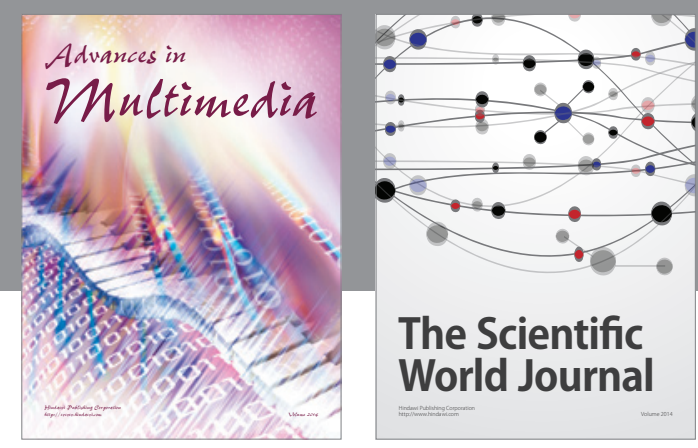

The Scientific World Journal
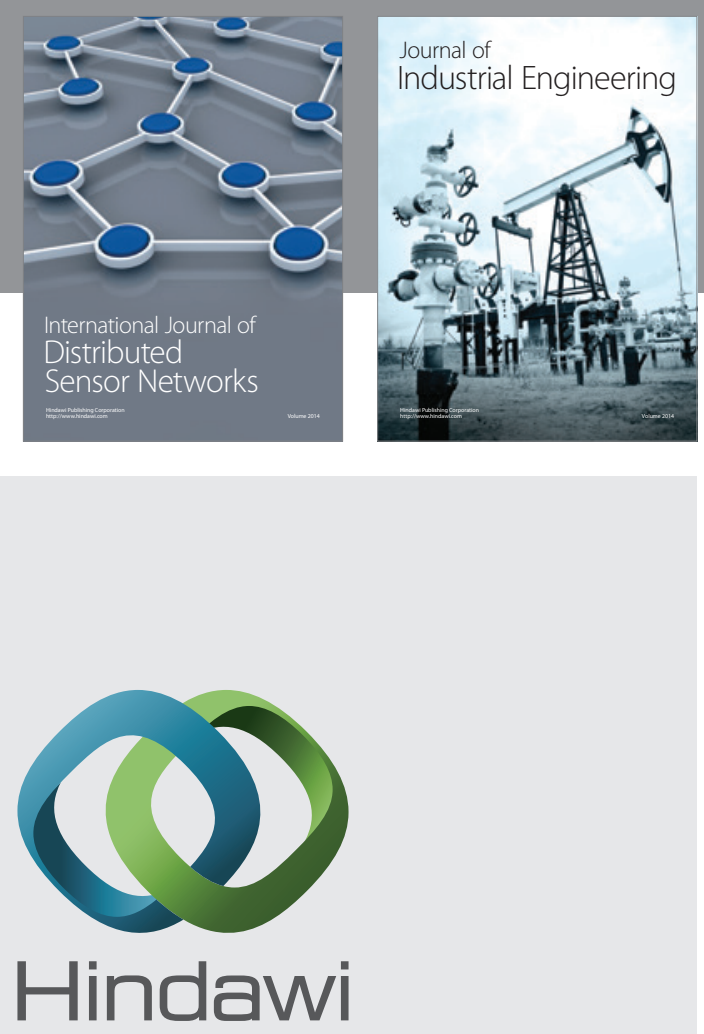

Submit your manuscripts at

https://www.hindawi.com

\section{Computer Networks} and Communications
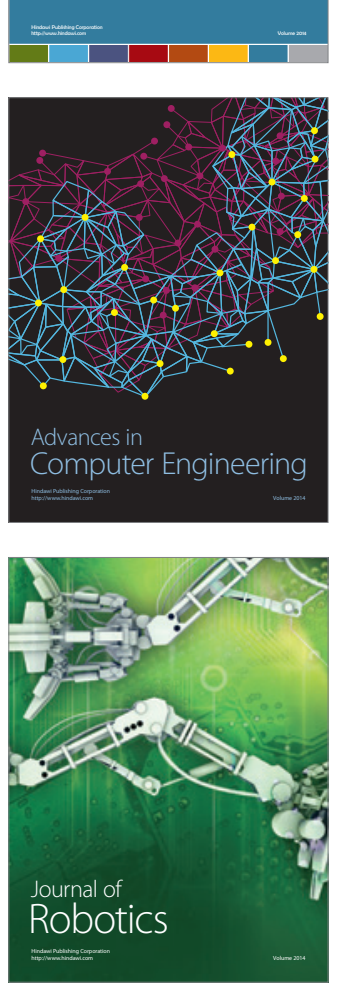
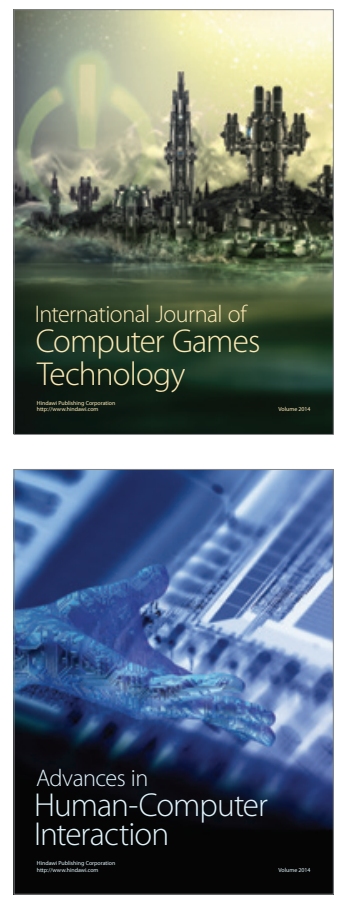
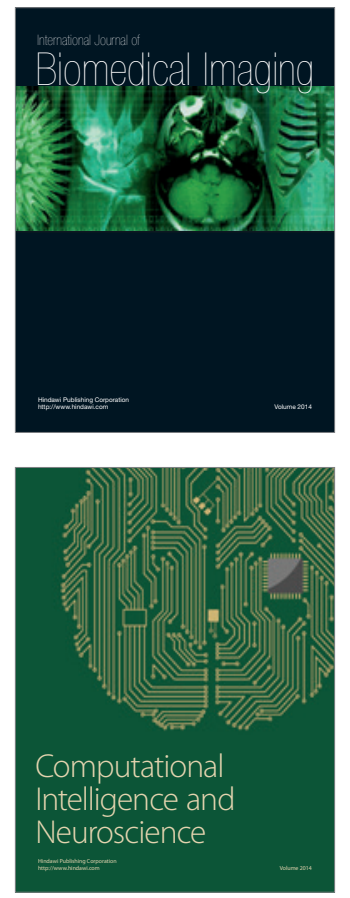
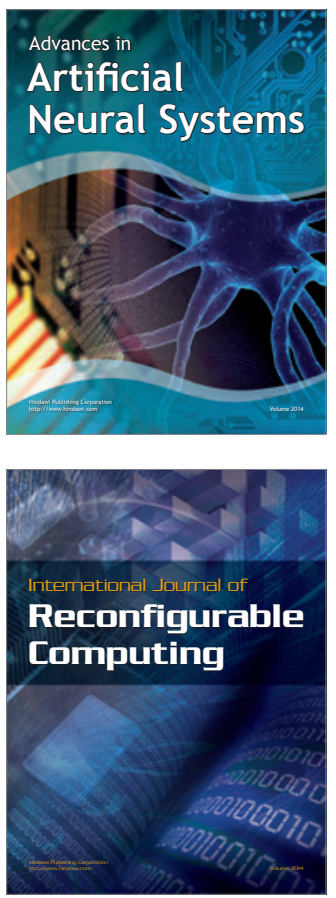
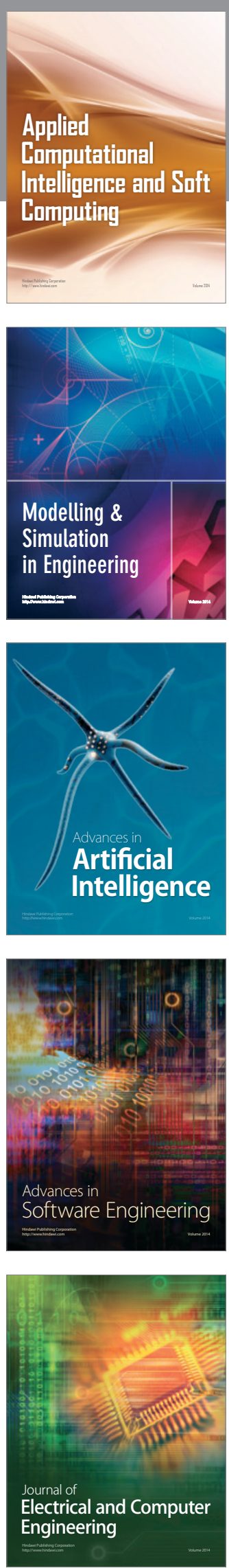